\title{
Phylogeny of three parapatric species of desert ants, Cataglyphis bicolor, C. viatica, and C. savignyi: A comparison of mitochondrial DNA, nuclear DNA, and morphological data
}

\author{
Markus Knaden ${ }^{\mathrm{a}, \mathrm{x}}$, Alberto Tinaut ${ }^{\mathrm{b}}$, Xim Cerda $^{\mathrm{c}}$, Sibylle Wehner ${ }^{\mathrm{a}}$, Rudiger Wehner ${ }^{\mathrm{a}}$ \\ a Department of Zoology, University of Zürich, Winterthurerstrasse 190, CH-8057 Zürich, Switzerland \\ ${ }^{\mathrm{b}}$ Departamento de Biologia Animal, Universitario de Fuantenueva, E-18071 Granada, Spain \\ ${ }^{\mathrm{c}}$ Departamento de Biologia Evolutiva, CSIC, Avenida de Maria Luisa, E-41013 Sevilla, Spain
}

\begin{abstract}
Due to morphological comparisons the Tunisian desert ant species Cataglyphis bicolor has been divided into three parapatric species: C. bicolor, C. viatica, and C. savignyi. The species status of the latter is supported by sequence analyses of the mitochondrial $\mathrm{CO} 1$ and $\mathrm{CO} 2$ region, while analyses of the same mitochondrial region lacked resolution for the separation of C. bicolor and C. viatica. However, the geographic distribution of mtDNA haplotypes points to different population viscosities with C. bicolor queens having longer migration distances than queens of C. viatica. Furthermore, by the use of microsatellites we excluded ongoing gene flow between geographically overlapping populations of $\mathrm{C}$. bicolor and C. viatica, and hence support the morphology-based three-species hypothesis. Concerning the ongoing discussion on the future roles of morphology and molecular biology in systematics we call for a combination of both whenever possible.
\end{abstract}

r 2005 Elsevier GmbH. All rights reserved.

Keywords: Ants; Cataglyphis; Male genitalia; MtDNA; Microsatellite; Taxonomy; Phylogeography

\section{Introduction}

North African desert ants of the genus Cataglyphis have become a model system for the study of animal navigation and the neurobiological mechanisms mediating visually guided behavior (Wehner, 2003). They have also been studied in ecological (Schmid-Hempel, 1983; Wehner et al., 1983; Dietrich and Wehner, 2003) and biogeographical terms (Wehner et al., 1994; for taxo-

\footnotetext{
${ }^{\times}$Corresponding author.

E-mail address: mknaden@zool.unizh.ch (M. Knaden).
}

nomic and biogeographical studies on Cataglyphis species of the Iberian peninsula see also Tinaut, 1990a, b). In the most recent study of Cataglyphis, Wehner et al. (1994) hypothesized that the classical species C. bicolor (sensu Santschi, 1929) should be divided into three parapatric species: C. viatica inhabiting the Mediterranean zone characterized by sclerophyllous shrub-like and low-grass vegetation, C. bicolor occupying the central Tunisian lowland and highland steppes, and C. savignyi living in the most foodimpoverished presaharan semi-deserts of southern Tunisia. In the present account, we investigate whether this splitting of C. bicolor into three separate species is 
supported by mitochondrial DNA data and microsatellite data, and how the molecular evidence correlates with comparative morphological characters of these three putative species.

\section{Material and methods}

During a transect we collected ants from the three putative species at the site localities indicated in terms of geographical latitude and longitude in Figs. 1 and 2. At each site we collected three worker specimens from one colony each. Due to the polydomy of several Cataglyphis species, we collected only from one colony per site to exclude double sampling of two nests from the same colony. The specimens were taken from the nest entrances. On the basis of the morphological characteristics described in Wehner et al. (1994) the specimens were assigned to one of the three putative species. They were stored in absolute ethanol until time of DNA extraction. Only one ant per nest was used, while the remaining two were reserved as voucher specimens. For a better resolution of the phylogeographic structure of

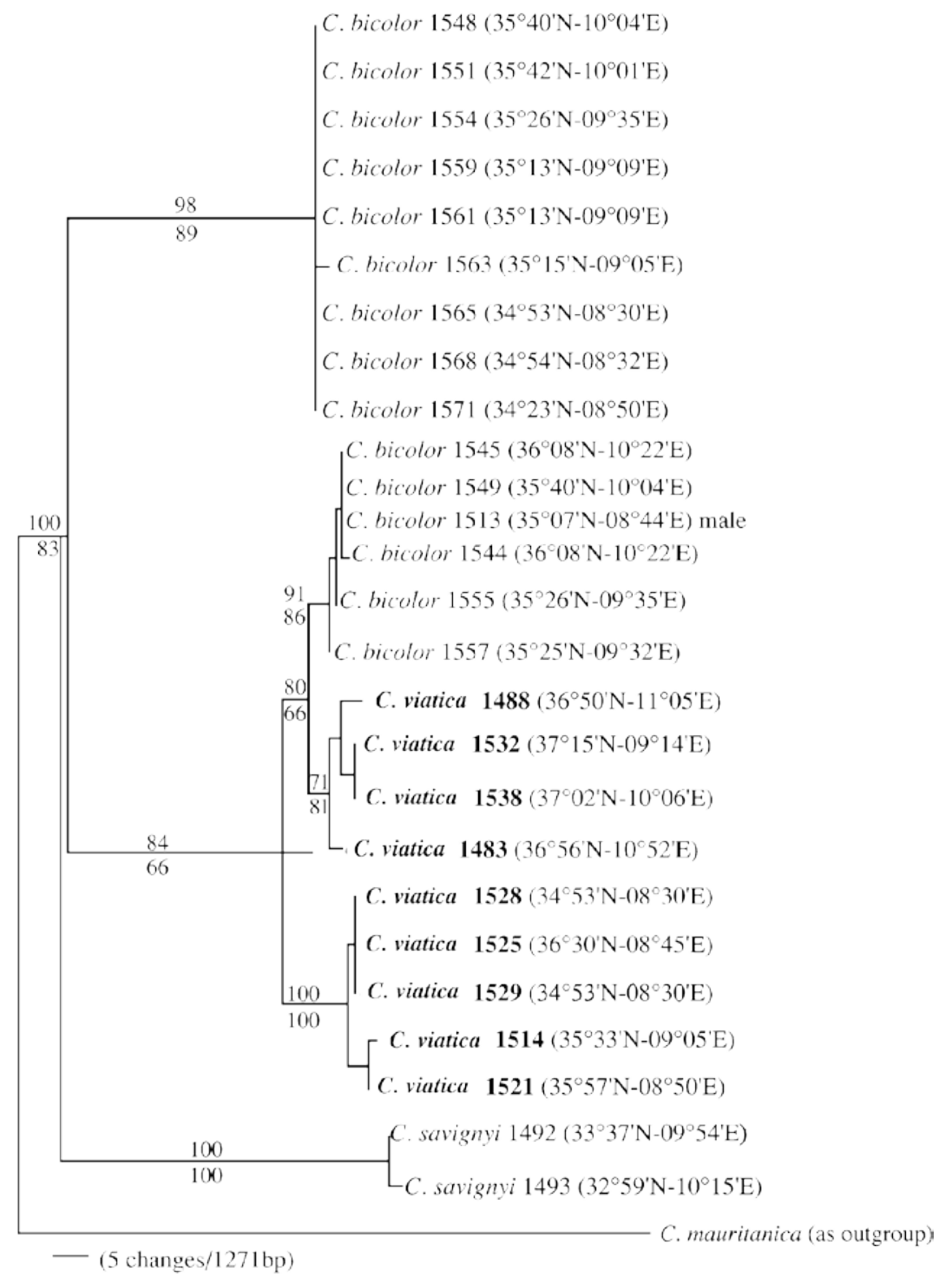

Fig. 1. Phylogram of C. bicolor, C. viatica, and C. savignyi inferred from mtDNA data. C. mauritanica (belonging to the altisquamis species group) is used as an outgroup. All nodes correspond with the bootstrap trees revealed by maximum parsimony (above nodes) and maximum likelihood (below nodes). Hence, bootstrap values for both maximum parsimony and maximum likelihood are given on the topology presented. The numbers of the bootstrap replicates are 2000 (100) and the numbers of the heuristic search are 100 (50) for maximum parsimony (maximum likelihood). Out of 1271 bp 1063 characters are constant, 103 are parsimony uninformative, and 105 are informative. Sample numbers refer to the collection code of the R. and S. Wehner Cataglyphis collection. Geographical coordinates are given in brackets. 


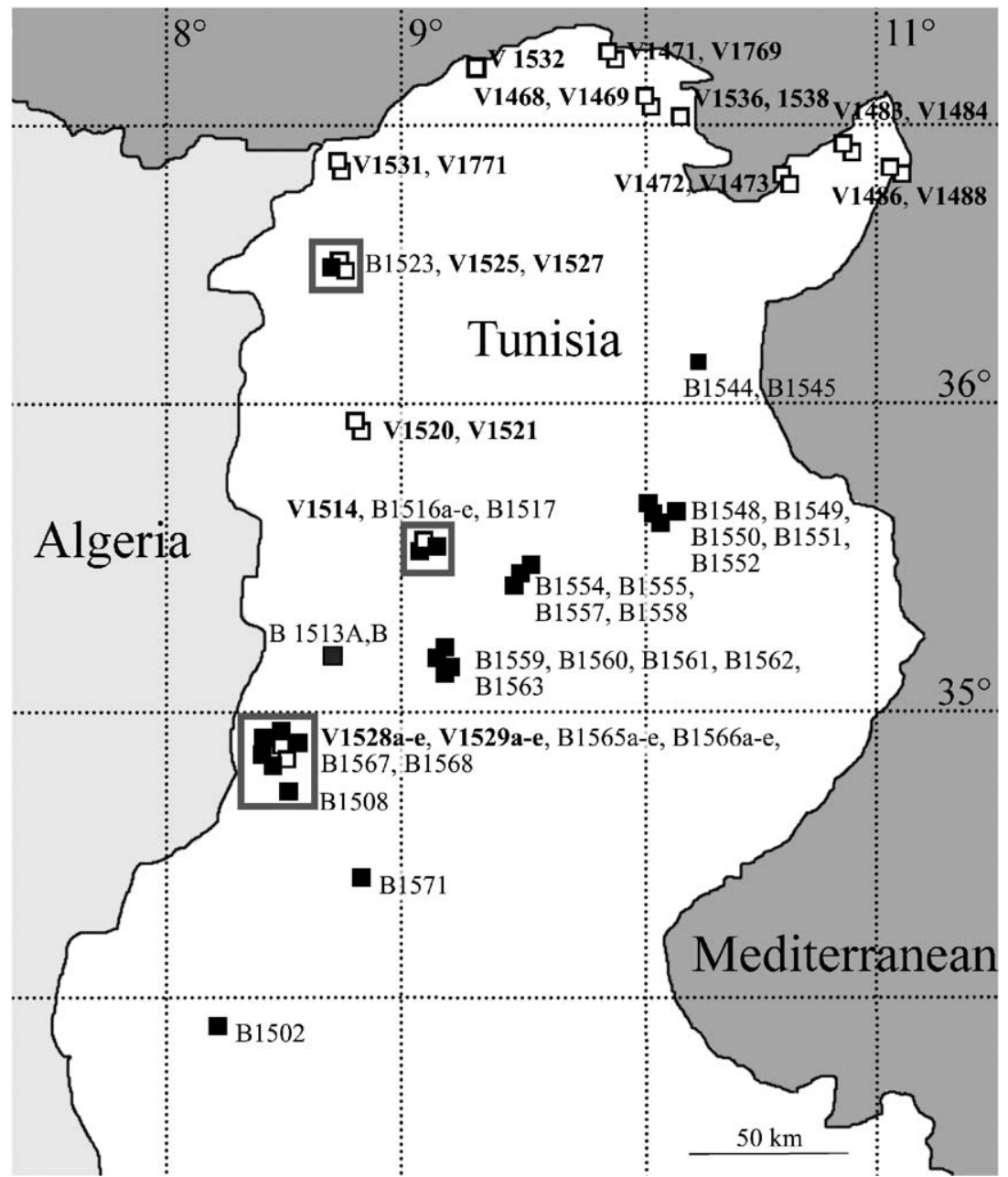

Fig. 2. Locations of the C. bicolor and C. viatica samples used in the mtDNA and microsatellite analyses. Filled black squares, locations of C. bicolor; open black squares, locations of C. viatica; open gray rectangles, areas in which C. bicolor and C. viatica coexist. Ants taken from these areas were analyzed separately for allele frequencies (see Table 2). The sample numbers refer to the collection code, B: C. bicolor, V: C. viatica).

the C. viatica and C. bicolor populations we later added specimens that already existed in the R. and S. Wehner Cataglyphis collection. Unless otherwise noted we focused on ants belonging to the worker caste. We extracted DNA from single ants (without head and gaster) using the CTAB method (Sambrock et al., 1989) with minor modifications. Proteinase K $(20 \mathrm{mg} / \mathrm{ml})$ was used instead of mercaptoethanol.

\section{Sequencing and phylogenetic analysis}

For the analysis of mitochondrial DNA the $3^{0}$ end within the cytochrome oxidase 1 gene (CO1) was amplified using the primer COI-RLR (50-TTGATTTTTTGGTCATCCAGAAGT-30 , Roehrdanz, 1993). This corresponds to position 2492 in the complete honeybee mitochondrial genome (Crozier and Crozier, 1993). For the $5^{0}$ end within the cytochrome oxidase 2 gene $(\mathrm{CO} 2)$ we used the primer Croz-COII (50-CCACAAATTTCTGAACATTGACC- $3^{0}$ ), which together with COI-RLR amplifies a sequence of about $1520 \mathrm{bp}$ including the leucine tRNA and an intergenic spacer. To enhance the sequence reaction in the inner part of the region we designed an internal primer pair COIF2 ( $5^{0}$-GCYAGATTCATTCATTGATTTCCTC- ${ }^{0}$, position 2929) and COIIR1bic (50-TGGGAGAATTTGAATTTTGAAGTG- $3^{0}$ ) amplifying 500 of the internal base pairs.

Polymerase chain reaction amplifications were carried out in $50 \mathrm{ml}$ reaction volumes containing 1 -Buffer A, $0.5 \mathrm{ml}$ DMSO, $0.2 \mathrm{mM}$ of each dNTP, $10 \mathrm{pM}$ of each primer, about $50 \mathrm{ng}$ DNA, and 1 unit Taq (Promega) with a PTC 100 (MJ Research) for 40 cycles (941C, 75 s, $431 \mathrm{C}, 75 \mathrm{~s}, 721 \mathrm{C}, 135 \mathrm{~s}$ ) after an initial $180 \mathrm{~s}$ denaturation step at $951 \mathrm{C}$ and with final extension at $721 \mathrm{C}$ for $300 \mathrm{~s}$. PCR reaction products were purified with the QIAquick PCR Purification Kit (Qiagen) under 
conditions specified by the manufacturer. PCR products were sequenced using the ABI-PRISM Dye Terminator Cycle Sequencing Ready Reaction Kit (ABI-Perkin Elmer) in $10 \mathrm{ml}$ reaction volumes following the manufacturer's instructions and run on an ABI 3100 DNA sequencer.

Chromatograms were first checked by eye for base call accuracy and then aligned individually with the opposite strand from the same individual using the program Sequencher ${ }^{\mathrm{TM}}$ (Gene Codes Corporation), and sequences were examined for sequence agreement. Finally, all sequences were checked for internal stop codons to exclude possible pseudogenes from analysis. All sequences were submitted to GenBank (Table 1).

Phylogenetic analyses of aligned sequences were carried out using the program PAUP*4.0b10 (Swofford, 2001). Due to the extensive length polymorphism of the intergenic spacer (C. viatica: 70-94 bp, C. bicolor: 76-106 bp, C. savignyi: 103 bp) we could not find any satisfying alignment. Furthermore, the position of the primer COIIR1 very close to the leucine tRNA gene resulted in less precise sequencing of this gene. Hence,

Table 1. List of material examined in the present study

\begin{tabular}{lll}
\hline Specimen (collection code) & \multicolumn{2}{l}{ GenBank nos. } \\
\cline { 2 - 3 } & CO1 & CO2 \\
\hline C. bicolor (1548) & AY737780 & AY737787 \\
C. bicolor (1551) & AY642288 & AY642296 \\
C. bicolor (1554) & AY737781 & AY737788 \\
C. bicolor (1559) & AY737782 & AY737789 \\
C. bicolor (1561) & AY737783 & AY737790 \\
C. bicolor (1565) & AY737784 & AY737791 \\
C. bicolor (1568) & AY737785 & AY737792 \\
C. bicolor (1571) & AY737786 & AY737793 \\
C. bicolor (1563) & AY642290 & AY642298 \\
C. bicolor (1513) & AY642289 & AY642297 \\
C. bicolor (1545) & AY642291 & AY642299 \\
C. bicolor (1549) & AY642292 & AY642300 \\
C. bicolor (1557) & AY642293 & AY642301 \\
C. bicolor (1544) & AY642294 & AY642302 \\
C. bicolor (1555) & AY642295 & AY642303 \\
C. savignyi (1492) & AY642304 & AY642306 \\
C. savignyi (1493) & AY642305 & AY642307 \\
C. viatica (1488) & AY642308 & AY642317 \\
C. viatica (1528) & AY642309 & AY642318 \\
C. viatica (1483) & AY642310 & AY642319 \\
C. viatica (1514) & AY642311 & AY642320 \\
C. viatica (1521) & AY642312 & AY642321 \\
C. viatica (1525) & AY642313 & AY642322 \\
C. viatica (1529) & AY642314 & AY642323 \\
C. viatica (1532) & AY642315 & AY642324 \\
C. viatica (1538) & AY642316 & AY642325 \\
C. mauritanica (1411) & AY642326 & AY642327 \\
& & \\
& & \\
& &
\end{tabular}

for calculating phylogenetic trees the non-coding region and the neighboring leucine tRNA were excluded. Maximum-parsimony trees inferred from either the $\mathrm{CO} 1$ region $(771 \mathrm{bp})$ or the $\mathrm{CO} 2$ region (500 bp) did not reveal contradicting nodes but revealed a higher resolution when $\mathrm{CO} 2$ sequences were used. There was a slightly lower variability within the CO1-coding region than in the CO2-coding region with a maximum sequence divergence of $1.04 \%$ (CO2: $2.40 \%$ ) within the $15 \mathrm{C}$. bicolor ants, $1.56 \%$ (CO2: $2.4 \%$ ) within the $9 \mathrm{C}$. viatica ants and $0.26 \%$ (CO2: $0 \%)$ in the $2 \mathrm{C}$. savignyi ants (partition homogeneity test: $\mathrm{p}^{1 / 4}$ 0:75).

Nevertheless, in the analyses both genes were combined. We found a linear correlation between substitutions at the third codon position and all substitutions as well as between transitions and all substitutions. Hence, saturation could be neglected, and for maximumparsimony analysis all substitutions were given equal weight. Heuristic searches were undertaken using the stepwise addition option with 100 random repetitions, TBR rearrangements and holding a maximum of 2000 trees. Clade support was assessed using 2000 bootstrap replicates (Felsenstein, 1985).

The most appropriate model of DNA substitution for maximum likelihood (ML) analyses was assessed using hierarchical likelihood ratio tests among a variety of competing models as implemented in the program Modeltest version 3.06 (Posada and Crandall, 1998). Because of the number of taxa involved and the computational time requirement, clade support for the best-fitting tree from ML analyses was assessed using 100 bootstrap replicates. We used C. mauritanica as an outgroup. This species belongs to a different Cataglyphis species group (altisquamis Group, Agosti, 1990).

\section{Comparative morphology data}

Male ants were taken from the $\mathrm{R}$. and S. Wehner Cataglyphis collection. Male genitalia were dissected and drawn using a binocular microscope (Wild M3B) with 40-fold extension (Fig. 3). We dissected 20 males of C. bicolor and 12 males of C. viatica from both the sympatric populations and the allopatric populations. Unfortunately most of these males were collected a long time ago (preserved in 70\% ethanol) and therefore only 4 C. bicolor males could be used for both the morphological and the mtDNA analyses.

\section{Microsatellite analysis}

For the analysis of nuclear DNA we added specimens from the site localities depicted in Fig. 2. We used the microsatellite primer pairs FE19 (50-GGACAAAGAAACAAGGAACG- $3^{0}$ and $5^{0}$-GATTTAGTTGGCTG- 
A

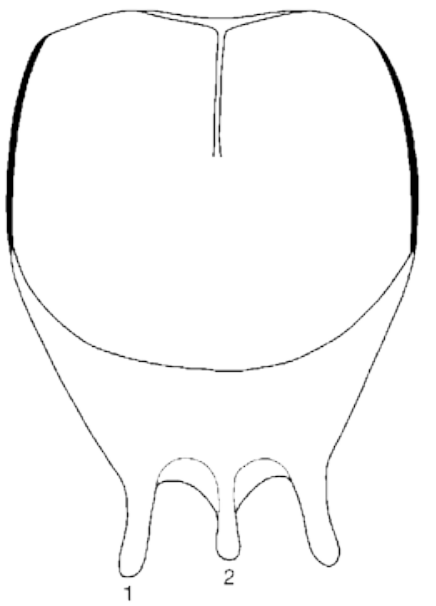

$\mathrm{B}$

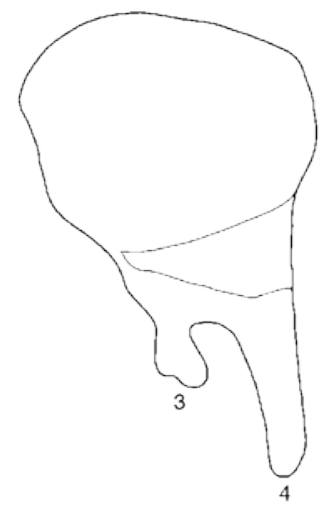

C

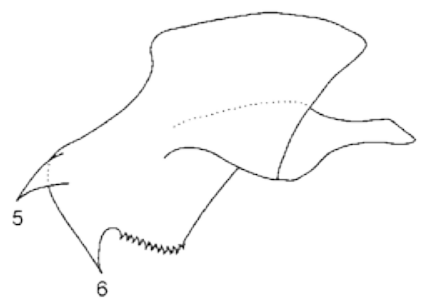

D

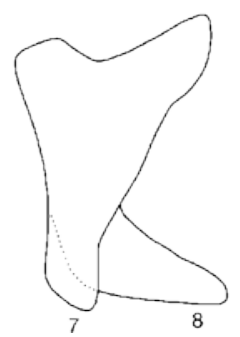

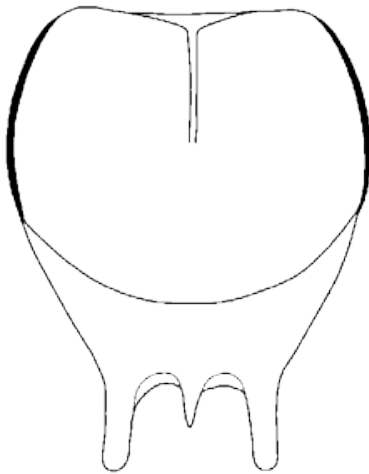
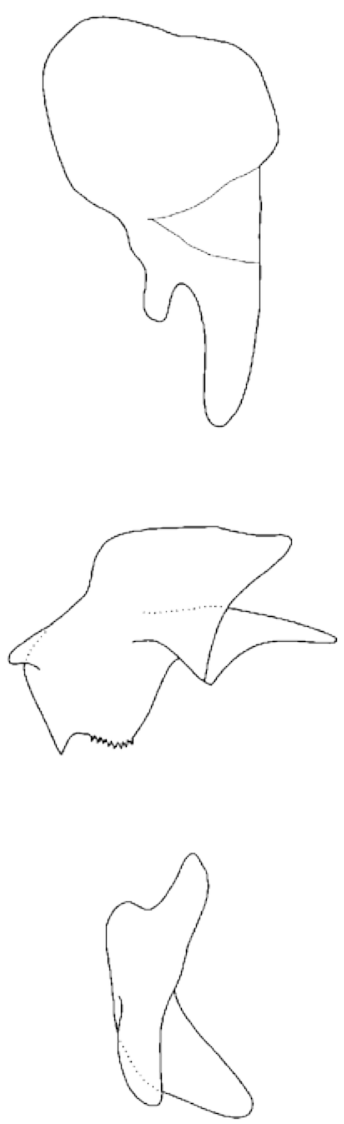

Fig. 3. Male genitalia in C. bicolor (left) and C. viatica (right). (A) Subgenital plate, inside view: lateral (1) and medial (2) processes. (B) Squamula: median appendix (3) and stipes (4). (C) Sagitta with apical process (5) and distal tooth (6). (D) Lacinia (7) and volsella (8). (B-D) are external views of the right-hand genitalia.

ACACGG-3 ${ }^{0}$ ) and FL29 (50-ATTGAGGAAGGCGGTGTTAC-30 and 50-CTTTAACGTATACCGTGCGG$3^{0}$ ), which were originally designed for Formica exsecta (Gyllenstrand et al., 2002) and F. lugubris (Chapuisat, 1996), respectively. Both amplify a TC/AG dinucleotide motif. PCR amplifications were carried out in $50 \mathrm{ml}$ as described above, but for 35 cycles with 951C, 60 s, 551C,
$60 \mathrm{~s}$, and $721 \mathrm{C}, 60 \mathrm{~s}$ again after initial $180 \mathrm{~s}$ at $951 \mathrm{C}$ and with subsequent final extension at $721 \mathrm{C}$ for $300 \mathrm{~s}$. PCR amplifications were analyzed on an EL 400 Wide Mini S-100 agarose gel (Elchrom) following the manufacturer's instructions. The allele sizes for each locus were determined and numbered according to their length (with allele 1 being the smallest). 


\section{Results}

\section{Phylogenetic analysis}

Out of 1271 bp sequenced 1063 characters were constant, 103 were parsimony uninformative, and 105 were informative. The sequences were tested for the most appropriate model of DNA substitution for maximum likelihood analyses by the program Modeltest version 3.06 (Posada and Crandall, 1998). The model GTR $+\mathrm{G}$ (Rodriguez et al., 1990) was the one that fit the data best. ML resulted in a tree with a -Ln likelihood of 2740.37344. Maximum parsimony resulted in only one tree with a tree length of 235. Both phylogenetic analyses performed resulted in identical tree topologies (Fig. 1). The mitochondrial sequence analysis clearly separated C. savignyi from the two other putative species (Fig. 1). In contrast, C. bicolor is paraphyletic with two well supported clades of which one is more closely related to $\mathrm{C}$. viatica. Therefore mtDNA CO1 and $\mathrm{CO} 2$ sequences, which have commonly been used in phylogenetic species-level analyses, are not sensitive enough to discriminate between C. bicolor and C. viatica.

In contrast to the purely phylogenetic analysis, C. bicolor and C. viatica seemed to differ in their phylogeographic population structure. We therefore included additional C. bicolor and C. viatica specimens in the analysis. Due to technical problems with these older samples, we were able to sequence only $771 \mathrm{bp}$ of the $\mathrm{CO} 1$ gene. One of the added $\mathrm{C}$. bicolor specimens clustered with the formerly monophyletic C. viatica clade and its sequences were even identical with those of four C. viatica ants (Fig. 4).

However, in contrast to the low resolution of the mtDNA analysis, C. bicolor and C. viatica showed different phylogeographic patterns. While the two clades that mainly consisted of C. viatica represent

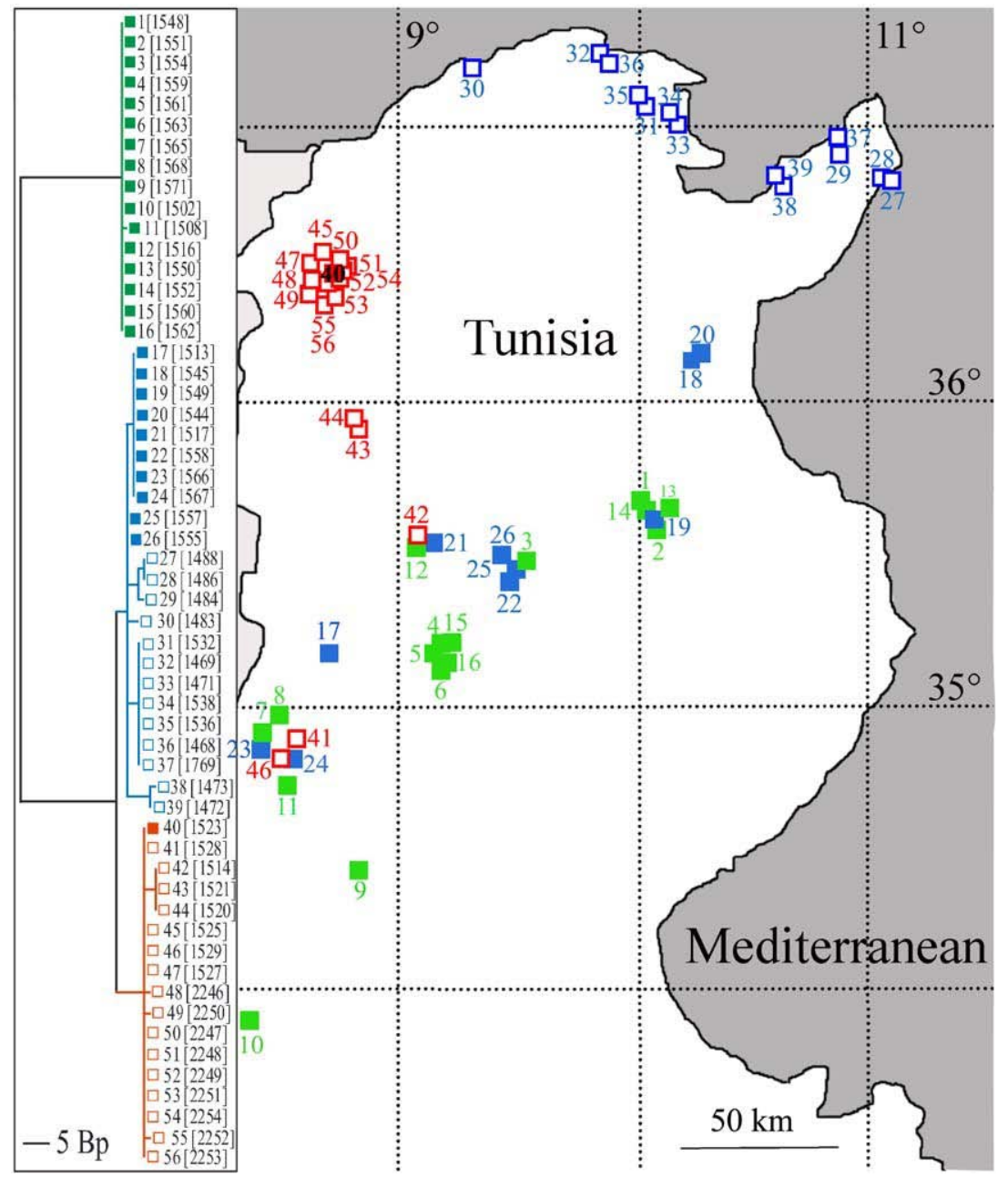

Fig. 4. Genetic population structure of C. bicolor and C. viatica inferred from mtDNA data. Filled squares, C. bicolor; open squares, C. viatica. Colors of the squares indicate to which main clade of the phylogram (left part of figure) the samples belong. The phylogram depicts the number of exchanged base pairs between the samples. Sample numbers in square brackets refer to the collection code of the R. and S. Wehner Cataglyphis collection. 
geographically distant populations in the northeast and northwest of Tunisia, the two clades containing C. bicolor are both spread over the whole distributional range of this species in Tunisia (Fig. 4).

\section{Comparative morphology analysis}

Morphological data obtained from the male genitalia, which in Cataglyphis comprise a highly distinctive set of characters (Arnoldi, 1964; Wehner et al., 1983; Agosti, 1990), are able to clearly separate C. viatica and C. bicolor (Fig. 3) and hence support the former conclusions (Wehner et al., 1994). Clear-cut differences between C. viatica and C. bicolor exist in all parts of the genitalian armature. The main distinctive features are the following (marked with numbers 1-8 in Fig. 3):

(a) Subgenital plate: The lateral finger-shaped processes (1) are slightly divergent and uniformly narrow from base to tip in C. bicolor, while in C. viatica they exhibit their smallest diameter in the middle and terminate slightly club-shaped. The central finger-shaped process (2) is short, narrow and pointed in C. viatica, while in C. bicolor it is longer (but shorter than the lateral processes) and terminates more bluntly.

(b) Squamula: In C. viatica the median appendix (3) inserts close to the stipes (4); in C. bicolor it is farther apart from the stipes, narrow at the base, and sometimes slightly cleft at the tip.

(c) Sagitta: The apical process (5) is more or less cylindrical in shape and rounded at the tip in C. viatica, but very pointed and heavily sclerotized in C. bicolor. The distal tooth (6) is triangular in shape and exhibits a wide base in C. viatica. In C. bicolor it is somewhat longer and its base is less wide than the length of the tooth.

(d) Lacinia (7) and volsella (8): In C. viatica the volsella is nearly straight and distinctly pointed at the tip. In C. bicolor its distal part is at an angle to its proximal part; apart from this, it is more uniform in shape than in C. viatica.

One of the four dissected C. bicolor males that could be sequenced belonged to the clade containing both
C. bicolor and C. viatica. Its genitalia did not differ from the three $\mathrm{C}$. bicolor individuals grouped in the other clade.

\section{Microsatellite analysis}

In order to examine whether C. viatica and C. bicolor are genetically isolated - even in those geographical regions in which both "species" coexist (Fig. 2) - we used microsatellite primers to test for gene flow. In the first locus (FL29) we found seven alleles in C. viatica ( $\mathrm{N}^{1 / 4} 30$ ants from 22 different colonies) and only two alleles in C. bicolor ( $\mathrm{N}^{1 / 4} 38$ ants from 25 different nests). The second locus exhibited 5 alleles in C. viatica, and only 3 alleles in C. bicolor (Table 2). Using the Gbased exact test (Goudet et al., 1996) which can be run at the GENEPOP website (Raymond and Rousset, 1995) we could reject the null hypothesis of allelic homogeneity between both populations (Markov chain parameters: dememorization: 1000, batches: 50, iterations per batch: 1000, po0:0001). This difference also applied to specimens of C. viatica and C. bicolor which occurred sympatricly (pO0:0001).

The observation that even overlapping populations of C. bicolor and C. viatica dramatically differ in their allele frequencies demonstrates the strongly restricted gene flow between them.

\section{Discussion}

When comparing morphology data (male genitalia) and mtDNA data (1271 bp of the CO1 and CO2 genes) in the three parapatric Tunisian Cataglyphis species, C. viatica, C. bicolor, and C. savignyi, one is confronted with contradictory data. All three species can be clearly separated by distinct morphological characters of males as well as workers (Wehner et al., 1994), but in the mtDNA analysis, C. viatica and C. bicolor cannot be disentangled. On the other hand, mtDNA and morphology data clearly separate $C$. savignyi from the other two "species". This is in accordance with a large-scale systematic analysis including 21 Cataglyphis species, where C. savignyi grouped closer to C. nodus, C. niger

Table 2. Allele frequencies in C. bicolor and C. viatica

\begin{tabular}{lcccccc}
\hline Alleles (FL29) & 1 & 2 & 3 & 4 & 5 & 7 \\
C. bicolor [72, 40]* & $88(78)$ & $12(22)$ & 0 & 0 & 0 & 0 \\
C. viatica [60, 30]* & $5(3)$ & $22(13)$ & $20(30)$ & $25(17)$ & $23(30)$ & $3(3)$ \\
Alleles (FE19) & 1 & 2 & 3 & 4 & 5 & $2(4)$ \\
C. bicolor [39, 12]* & 0 & $10(0)$ & $87(92)$ & $3(8)$ & 0 & \\
C. viatica [36,14]* & $3(0)$ & $42(21)$ & $25(29)$ & $25(43)$ & $5(7)$ &
\end{tabular}

Numbers: frequencies in the whole populations of C. bicolor and C. viatica; numbers in brackets: frequencies in the sympatric populations. *First number: sample sizes taken from the total population, second number: sample sizes taken from the sympatric populations. 
and C. abyssinicus than to C. bicolor (Knaden et al., unpublished data).

Hence the question arises: are C. viatica and C. bicolor really distinct species - or, in other words, why do the results of the mtDNA analysis and of the morphological analyses give different pictures? A closer look at the clade containing $\mathrm{C}$. bicolor and $\mathrm{C}$. viatica shows that both are somewhat distinct, forming two minor clades (Fig. 1). The paraphyletic emergence of C. bicolor (Fig. 1) could even mean that the three-species hypothesis underestimates the number of distinct species, with the putative C. bicolor forming two separate taxonomic categories.

However, if the phylogeographic patterns of the different C. bicolor and C. viatica clades are taken into account, the two C. viatica clades are clearly separated geographically. They occupy different areas in the north-eastern and north-western parts of the distributional range described for C. viatica (cf. Fig. 7 in Wehner et al., 1994). On the other hand, there was no obvious phylogeographic structure in the clades containing C. bicolor (Fig. 4). Due to the monogyny of both C. bicolor and C. viatica (Wehner et al., 1994) such differences in population viscosities are unexpected. Usually queens of monogynous ants perform far reaching mating flights (Keller, 1991) that should prevent a population from being genetically structured in the way C. viatica apparently is.

Interestingly, the single $\mathrm{C}$. bicolor that grouped on the C. viatica clade came from the outermost northern border of the distributional range of $\mathrm{C}$. bicolor, where C. bicolor and C. viatica co-occur. It had the same mtDNA haplotype as some of the $C$. viatica ants collected close-by. This might be a hint for an, albeit rare, hybridization between $\mathrm{C}$. bicolor and $\mathrm{C}$. viatica.

A re-evaluation of the morphological characters of the male genitalia which have been used in phylogenetic reconstructions in formicine ants in general (Bolton, 1990; Ward, 1990; BaroniUrbani et al., 1992; Andrade and BaroniUrbani, 1999), and in Cataglyphis species in particular (Agosti, 1990; Tinaut, 1990a, b), supported the former conclusion (Wehner et al., 1994) that C. viatica and C. bicolor are clearly separated species. Furthermore, we could not find any differences in the male genitalia of the two well-supported clades of C. bicolor. The solution to the $\mathrm{C}$. viatica/C. bicolor problem came when we complemented our mtDNA analysis with microsatellite comparisons. This allowed us to test for interbreeding between C. viatica and C. bicolor in those geographical regions in which their distributional ranges overlap. C. bicolor revealed less variability in both of the tested loci (FL29: 2 versus 7 alleles in C. viatica, FE19: 3 versus 5 alleles in C. viatica). Although the single $\mathrm{C}$. bicolor grouping on the C. viatica clade in the mtDNA analysis might point to an event of hybridization in the past, due to the large differences between the allele frequencies the result was clear-cut: there is no indication of ongoing gene flow between the two populations of, hence, heterospecific Cataglyphis ants.

Being paraphyletic when only mtDNA data are used, C. bicolor might form one ore two separate taxonomic categories. However, the result that C. bicolor and C. viatica cannot be separated at the mtDNA level may also indicate a very recent divergence of the two species which have not yet undergone the stochastic process of complete lineage sorting.

Sequence analysis of $\mathrm{CO} 1$ and $\mathrm{CO} 2$ is used extensively in phylogenetic analyses (CO1: Leys et al., 2000; Tanaka et al., 2001; Colgan et al., 2003; CO2: Normark, 2000; Salvato et al., 2002). The separation of C. savignyi from the other two species included in this analysis as well as the results from preliminary analyses of additional Cataglyphis species (Knaden et al., unpublished data) reflects the taxonomic power of the $\mathrm{CO} 1-\mathrm{CO} 2$ analyses. In northern Tunisia there are some populations of C. bicolor and C. viatica that occur sympatrically, allowing us to test for genetic isolation among populations. The lack of gene flow between these populations supports the conclusion based on the morphological studies. Exhibiting clear morphological differences and showing no actual gene flow in sympatric populations, the speciation hypothesis for C. bicolor and C. viatica is in agreement with the biological species concept (Mayr, 1942).

There is an ongoing discussion on the future roles of morphology and molecular biology in taxonomy (Godfray, 2002; Lipscomb et al., 2003; Seberg et al., 2003; Tautz et al., 2003). In C. bicolor and C. viatica the morphological separation was supported by the microsatellite analyses, while the missing resolution within the mtDNA tree hints at a rather recent separation of the two species. Hence, it would be advantageous if further discussions did not focus on benefits and handicaps of morphology and molecular biology, but led to a fruitful combination of both approaches.

\section{Acknowledgments}

We thank Robyn Tourle for help in the line drawings of the male genitalia. Financial support came from the Swiss National Science Foundation (Grant No. 3161844.0), the G. and A. Claraz Foundation, and the Promoter Foundation (all to RW).

\section{References}

Agosti, D., 1990. Review and reclassification of Cataglyphis (Hymenoptera, Formicidae). J. Nat. Hist. 24, 1457-1505. 
Andrade, M.L., Baroni Urbani, C., 1999. Diversity and adaptation in the ant genus Cephalotes, past and present. Stuttg. Beitr. Naturkd. B 271, 1-889.

Arnoldi, K.W., 1964. The highly specialized species of Cataglyphis (Hymenoptera, Formicidae) in Russia. Zool. Z. 43, 1800-1814 [in Russian].

Baroni Urbani, C., Bolton, B., Ward, P.S., 1992. The internal phylogeny of ants (Hymenoptera: Formicidae). Syst. Entomol. 17, 301-329.

Bolton, B., 1990. Army ants reassessed: the phylogeny and classification of the dorylinae section (Hymenoptera, Formicidae). J. Nat. Hist. 24, 1339-1364.

Chapuisat, M., 1996. Characterization of microsatellite loci in Formica lugubris B and their variability in other ant species. Mol. Ecol. 5, 599-601.

Colgan, D.J., Cassis, G., Beacham, E., 2003. Setting the molecular phylogenetic framework for the Dermaptera. Ins. Syst. Evol. 34, 65-80.

Crozier, R.H., Crozier, Y.C., 1993. The mitochondrial genome of the honeybee Apis mellifera: complete sequence and genome organization. Genetics 113, 97-117.

Dietrich, B., Wehner, W., 2003. Sympatry and allopatry in two desert ant sister species: how do Cataglyphis bicolor and C. savignyi coexist? Oecologia 136, 63-72.

Felsenstein, J., 1985. Confidence limits on phylogenies: an approach using bootstrap. Evolution 39, 783-791.

Godfray, H.C.J., 2002. Challenges for taxonomy. Nature 417, 17-19.

Goudet, J., Raymond, M., De Meeus, T., Rousset, F., 1996. Testing differentiation in diploid populations. Genetics 144, 1933-1940.

Gyllenstrand, N., Gertsch, P.J., Pamilo, P., 2002. Polymorphic microsatellite DNA markers in the ant Formica exsecta. Mol. Ecol. 2, 67-69.

Keller, L., 1991. Queen number, mode of colony founding, and queen reproductive success in ants (Hymenoptera: Formicidae). Ethol. Ecol. Evol. 3, 307-316.

Leys, R., Cooper, S.J.B., Schwarz, M.P., 2000. Molecular phylogeny of the large carpenter bees, genus Xylocopa (Hymenoptera: Apidae), based on mitochondrial DNA sequences. Mol. Phyl. Evol. 17, 407-418.

Lipscomb, D., Platnick, N., Wheeler, Q., 2003. The intellectual content of taxonomy: a comment on DNA taxonomy. Trends Ecol. Evol. 18, 65-66.

Mayr, E., 1942. Systematics and the Origin of Species. Columbia University Press, New York.

Normark, B.B., 2000. Molecular systematics and evolution of the aphid family Lachnidae. Mol. Phyl. Evol. 14, 131-140.

Posada, D., Crandall, K.A., 1998. Modeltest: testing the model of DNA substitution. Bioinformatics 14, 817-818.

Raymond, M., Rousset, F., 1995. GENEPOP (version 1.2): population genetics software for exact tests and ecumenicism. J. Heredity 86, 248-249.

Rodriguez, F., Oliver, J.F., Marin, A., Medina, J.R., 1990. The general stochastic model of nucleotide substitution. J. Theor. Biol. 142, 485-501.
Roehrdanz, R.L., 1993. An improved primer for PCR amplification of mitochondrial DNA in a variety of insect species. Insect Mol. Biol. 2, 89-91.

Salvato, P., Battisti, A., Concato, S., Masutti, L., Patarnello, T., Zane, L., 2002. Genetic differentiation in the winter pine processionary moth (Thaumetopoea pityocampa-wilkinsoni complex) inferred by AFLP and mitochondrial DNA markers. Mol. Ecol. 11, 2435-2444.

Sambrock, J., Fritsch, E.F., Maniatis, T., 1989. Molecular Cloning. A Laboratory Manual. Cold Spring Harbor Laboratory Press, New York.

Santschi, F., 1929. Etudes sur les Cataglyphis. Rev. Suisse Zool. 36, 25-70.

Schmid-Hempel, P., 1983. Foraging ecology and colony structure of two sympatric species of desert ants, Cataglyphis bicolor and Cataglyphis albicans. Ph.D. Thesis. University of Zurich.

Seberg, O., Humphries, C.J., Knapp, S., Stevenson, D.W., Petersen, G., Scharff, N., Andersen, N.M., 2003. Shortcuts in systematics? A commentary on DNA-based taxonomy. TREE 18, 63-65.

Swofford, D.L., 2001. PAUP*: Phylogenetic Analysis Using Parsimony (and Other Methods), version 4. Computer Program distributed by Sinauer Associates, Sunderland, MA.

Tanaka, H., Roubik, D.W., Kato, M., Liew, F., Gunsalam, G., 2001. Phylogenetic position of Apis nuluensis of northern Borneo and phylogeography of A. cerana as inferred from mitochondrial DNA sequences. Insectes Sociaux 48, 44-51.

Tautz, D., Arctander, P., Minelli, A., Thomas, R.H., Vogler, A.P., 2003. A plea for DNA taxonomy. TREE 18, 70-74.

Tinaut, A., 1990a. Taxonomic situation of the genus Cataglyphis Forster 1850 in the Iberian peninsula II. New position for C. viatica (Fabricius, 1787) and redescription of C. velox Santschi, 1929 stat. n. (Hymenoptera, Formicidae). Eos 66, 49-59.

Tinaut, A., 1990b. Taxonomic situation of the genus Cataglyphis Forster 1850 in the Iberian peninsula III. The Cataglyphis velox Santschi 1929 group and description of Cataglyphis humeya sp. n. (Hymenoptera, Formicidae). Eos 66, 215-227.

Ward, P.S., 1990. The ant subfamily Pseudomyrmecinae (Hymenoptera: Formicidae): generic revision and relationship to other formicides. Syst. Entomol. 15, 449-489.

Wehner, R., 2003. Desert ant navigation: how miniature brains solve complex tasks (Karl von Frisch Lecture). J. Comp. Physiol. A 189, 579-588.

Wehner, R., Harkness, R.D., Schmid-Hempel, P., 1983. Foraging Strategies in Individually Searching Ants Cataglyphis bicolor (Hymenoptera: Formicidae). Akad. Wiss. Lit. Mainz, Gustav Fischer Verlag, Stuttgart.

Wehner, R., Wehner, S., Agosti, D., 1994. Patterns of biogeographic distribution within the bicolor species group of the North African desert ant, Cataglyphis Forster 1850 (Insecta: Hymenoptera: Formicidae). Senckenbergiana Biol. 74, 163-191. 